\title{
Dual Chamber Pacemaker
}

National Cancer Institute

\section{Source}

National Cancer Institute. Dual Chamber Pacemaker. NCI Thesaurus. Code C80459.

A heart pacemaker with the capability of monitoring and stimulating both the atria and/or the ventricles when necessary. 